\title{
Metabolic-associated Fatty Liver Disease: Time to integrate ground-breaking new terminology to our clinical practice?
}

\author{
Coskun Ozer Demirtas ${ }^{1}$, (D) Yusuf Yilmaz ${ }^{1,2}$ \\ ${ }^{1}$ Department of Gastroenterology, Marmara University, School of Medicine, Istanbul, Turkey; \\ ${ }^{2}$ Marmara University, Institute of Gastroenterology, Istanbul, Turkey
}

\begin{abstract}
Non-alcoholic fatty liver disease (NAFLD) was coined as a defined pathological entity in 1980 as a condition mimicking alcoholic fatty liver disease with excessive fat infiltration to the liver but in the absence of significant alcohol consumption or other causes of liver disease. ${ }^{[1]}$ Over the past 40 years, our understanding of NAFLD has evolved to broadly define a link to metabolic dysregulation as the driving force in the pathogenesis of the disease. Many reports highlighting its linkage to obesity, obesity-associated diseases and insulin resistance have been published. Since its original naming, NAFLD has been accepted as the standard acronym to describe this entity. Today, this condition has become the most common cause of liver disease globally. In Turkey, an alarming prevalence of $48.3 \%$ for the fatty liver disease has been reported, which is representative of the growing burden of obesity, type 2 diabetes mellitus and metabolic syndrome globally. ${ }^{[2]}$ With the increasing prevalence of the disease and advances in our understanding of it, several concerns and criticisms have been raised on the appropriateness of the term "NAFLD". Several alternative terminology attempts have been proposed to rename NAFLD, each with a different emphasis and varying degrees of reconceptualization. ${ }^{[3-6]}$ The common criticism points of these articles were that NAFLD reflects a negative term with use of the prefix "non-", the dominant role of "alcohol" in the term, and its inability to cover a highly heterogeneous group of patients under the NAFLD umbrella. In addition, the diagnostic criteria for NAFLD to define fatty liver disease associated with metabolic dysfunction are based solely on the exclusion of other conditions, such as alcohol use and/or viral hepatitis. Another major limitation with the traditional definition is that it prevents persons with traditional risk factors for NAFLD (e.g., central obesity and type 2 diabetes) who consume alcohol in excess of arbitrary thresholds to qualify for the diagnosis of NAFLD. ${ }^{[7,8]}$ However, in real-world clinical practice, diagnoses of alcoholic liver disease and NAFLD may overlap, and it is challenging to distinguish one from the other in such situations. Fur-
\end{abstract}

How to cite this article: Demirtas CO, Yilmaz Y. Metabolic-associated Fatty Liver Disease: Time to integrate ground-breaking new terminology to our clinical practice? Hepatology Forum 2020; 1(3):79-81.

Received: September 08, 2020; Accepted: September 09, 2020; Available online: September 21, 2020

Corresponding author: Yusuf Yilmaz; Marmara Universitesi, Gastroenteroloji Enstitusu, Maltepe, Istanbul, Turkey

Phone: +90 53344039 95; e-mail: dryusufyilmaz@gmail.com

(1) OP OPen access

c. This work is licensed under a Creative Commons Attribution-NonCommercial 4.0 International License.

(C) Copyright 2020 by Hepatology Forum - Available online at www.hepatologyforum.org thermore, the NAFLD terminology does not cover or indicate the key role of metabolic factors as significant pathogenetic drivers in its name nor in its definition.

To address these issues, an international expert panel consensus was recently reached by 32 distinguished experts from 22 countries to propose a more comprehensive redefinition of fatty liver disease associated with metabolic dysfunction. This includes a more apt name, "metabolic (dysfunction) associated fatty liver disease (MAFLD)" to replace NAFLD ${ }^{[9]}$ and an easily applicable set of "positive" diagnostic criteria. The diagnosis of MAFLD can be entertained in the presence of hepatic steatosis in addition to at least one of the following three criteria: 1) overweight/ obesity [body mass index in Caucasians (BMI) $\geq 25 \mathrm{~kg} / \mathrm{m}^{2}$ ], 2) presence of type 2 diabetes mellitus or 3 ) evidence of metabolic dysregulation defined by the presence of at least two of the seven metabolic at-risk criteria listed in Figure $1 .{ }^{[9,10]}$

The major advantage of the new definition is that it better reflects knowledge of the underlying metabolic pathophysiology that primarily drives MAFLD. By replacing NAFLD with MAFLD, the role of metabolic dysfunction becomes central, while alcohol is considered just one of the multiple potential factors that can exacerbate the disease. In fact, the definition classifies this disease as a metabolic disorder by not only having the word "metabolic" in its name but also requiring evidence of metabolic dysregulation, which was lacking in the old definition. The MAFLD terminology embraces the full spectrum of disease from simple steatosis with no inflammation and fibrosis to stage 4 fibrosis, including those with burnt-out steatosis. Therefore, the adoption of the umbrella term will likely to result in abandoning the simplistic dichotomization to NASH and non-NASH, moving instead to assessing disease severity that comprises both inflammation and fibrosis stage, akin to what is undertaken for all other liver diseases. From this perspective, the term MAFLD-related cirrhosis is expected to replace the old "cryptogenic cirrhosis" definition for the majority of patients. Furthermore, it avoids any reference to alcohol intake as an exclusion criterion. According to the new criteria, the exclusion of other liver diseases is not mandatory but would be required for a holistic assessment of patients in order to exclude contributions from other diseases.

By all accounts, to this clinician researcher, the "MAFLD" definition seems to be a more appropriate umbrella term to capture patients than the old "NAFLD" definition. Besides, the integration of "metabolic" in the name and the positive criteria used to diagnose patients is expected to reduce confusion on disease etiology, stigma and facilitate patient-physician communication. Despite these obvious benefits, for some, the name change is still viewed as premature and one that requires building a wider consensus. In this regard, the main issues ahead 


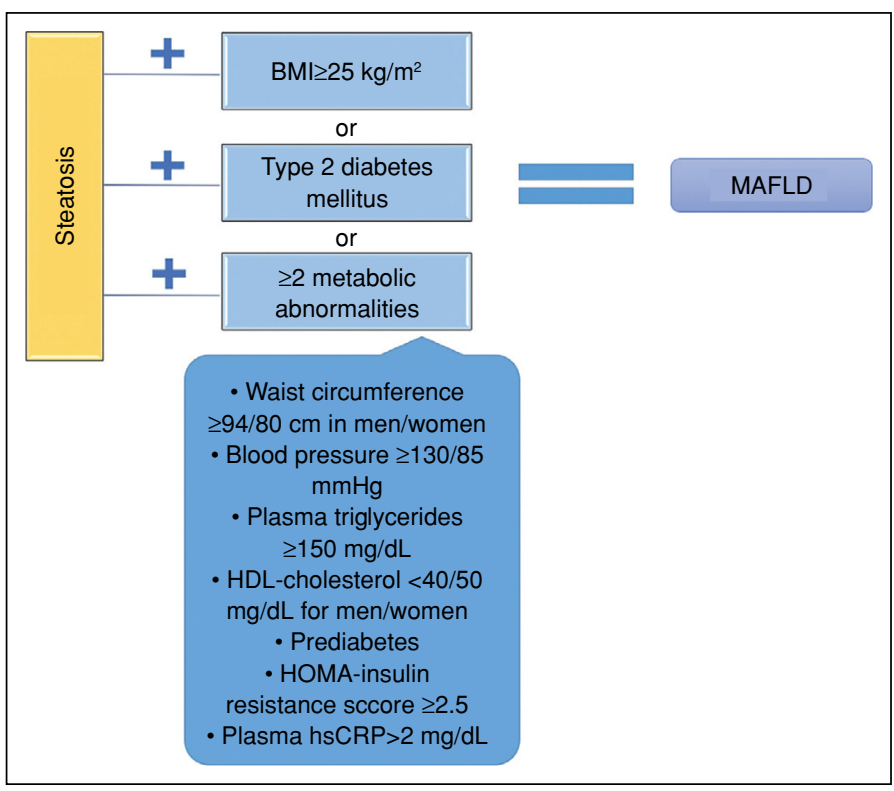

Figure 1. Diagnostic criteria of the MAFLD based on the new consensus BMI: Body-mass index; HDL: High-density lipoprotein; hsCRP: high-sensitive C-reactive protein; HOMA: Homeostatic model assessment; MAFLD: Metabolic-associated fatty liver disease.

include the need for further clarification and stratification of MAFLD to guide decision-making and prognostication. Some authors also criticize the new definition because it lacks molecular pathobiological integration; they suggest that we consider a name change only when informed by a new understanding of the molecular basis of the disease that would change aspects such as risk stratification. ${ }^{[11]}$ For instance, there is no information about phospholipase domain-containing protein-3 (PNPLA3) and other MAFLD-related genetic variants, especially in the subgroup of lean individuals without metabolic dysregulation. Indeed, these aspects support rather than refute the concept behind the redefinition. Deconvolution of heterogeneity in the population previously assembled under the term "NAFLD" is one of the main drivers for this new concept, as alluded too by the consensus experts. Indeed heterogeneity is likely to be responsible for the high rate of failure in NAFLD clinical trials and the suboptimal performance of non-invasive tests. From this perspective, it is equally possible that lean individuals without evidence of metabolic dysregulation should be considered a distinct entity with a distinct natural history and clinical outcomes. In turn, it suggests that MAFLD can overcome some of the inherent limitations of the patients characterised as having NAFLD. ${ }^{[12]}$ While patient sub-stratification is the ultimate goal, the new definition is a crucial and appropriate first step. Despite these concerns, it must be acknowledged that the new definition has attracted considerable attention from hepatologists, with many reports and editorials published acknowledging and supporting utilization of the MAFLD criteria. ${ }^{[13-18]}$ Moreover, in studies investigating the role of liver disease in the prognosis of COVID-19 (as a proxy for the recent submitted publications), the MAFLD definition has already been incorporated. ${ }^{[19-21]}$ More recently, two studies have suggested that the MAFLD criteria are more practical and identify patients at high risk of fibrosis better than the NAFLD criteria. ${ }^{[22,23]}$

From our point of view, it, therefore, seems to be reasonable to change the acronym from NAFLD to MAFLD considering the considerable benefits that will accrue for patients, public awareness and clinicians and allied health staff. The international expert consensus is but the start in the "MAFLD" journey; global endorsement by researchers, clinicians, industry and patients will take time. While discussions on the necessity for such a change continues, more research is required that is not only significant from an abstract theoretical perspective, but also concerning applicability and suitability for clinical practice, clinical management and clinical trials that support the global positive first impression of this new terminology.

\section{References}

1. Ludwig J, Viggiano TR, McGill DB, Oh BJ. Nonalcoholic steatohepatitis: Mayo Clinic experiences with a hitherto unnamed disease. Mayo Clin Proc 1980;55(7):434-438.

2. Yılmaz Y, Kanı HT, Demirtaş CÖ, Kaya E, Sapmaz AF, Qutranji L, et al. Growing burden of nonalcoholic fatty liver disease in Turkey: A single-center experience. Turk J Gastroenterol 2019;30(10):892-898.

3. Loria P, Lonardo A, Carulli N. Should nonalcoholic fatty liver disease be renamed? Dig Dis 2005;23(1):72-82.

4. Dufour JF. Time to Abandon NASH? Hepatology 2016;63(1):9-10.

5. Bellentani S, Tiribelli C. Is it time to change NAFLD and NASH nomenclature? Lancet Gastroenterol Hepatol 2017;2(8):547-548.

6. Eslam M, Sanyal AJ, George J. Toward More Accurate Nomenclature for Fatty Liver Diseases. Gastroenterology 2019;157(3):590-593.

7. Rinella ME, Loomba R, Caldwell SH, Kowdley K, Charlton M, Tetri B, et al. Controversies in the Diagnosis and Management of NAFLD and NASH. Gastroenterol Hepatol (N Y) 2014;10(4):219-227.

8. Perumpail BJ, Khan MA, Yoo ER, Cholankeril G, Kim D, Ahmed A. Clinical epidemiology and disease burden of nonalcoholic fatty liver disease. World J Gastroenterol 2017(47):8263-8276.

9. Eslam M, Sanyal AJ, George J; International Consensus Panel. MAFLD: A Consensus-Driven Proposed Nomenclature for Metabolic Associated Fatty Liver Disease. Gastroenterology 2020;158(7):1999-2014.e1.

10. Eslam M, Newsome PN, Sarin SK, Anstee QM, Targher G, Romero-Gomez $\mathrm{M}$, et al. A new definition for metabolic dysfunction-associated fatty liver disease: An international expert consensus statement. J Hepatol 2020;73(1):202-209.

11. Younossi ZM, Rinella ME, Sanyal A, Harrison SA, Brunt E, Goodman Z, et al. From NAFLD to MAFLD: Implications of a premature change in terminology. Hepatology 2020 Jun 16. doi: 10.1002/hep.31420. [Epub ahead of print].

12. Eslam M, George J. Reply to: Correspondence on "A new definition for metabolic associated fatty liver disease: an international expert consensus statement": MAFLD: Moving from a concept to practice. J Hepatol 2020:S0168-8278(20)30439-30436.

13. Huang J, Kumar R, Wang M, Zhu Y, Lin S. MAFLD criteria overlooks a number of patients with severe steatosis: Is it clinically relevant? J Hepatol 2020:S0168-8278(20)30387-30381.

14. Fouad Y, Waked I, Bollipo S, Gomaa A, Ajlouni Y, Attia D. What's in a name? Renaming 'NAFLD' to 'MAFLD'. Liver Int 2020;40(6):1254-1261.

15. The Lancet Gastroenterology Hepatology. Redefining non-alcoholic fatty liver disease: what's in a name? Lancet Gastroenterol Hepatol 2020;5(5):419.

16. Valenti L, Pelusi S. Redefining fatty liver disease classification in 2020. Liver Int 2020;40(5):1016-1017.

17. Zheng KI, Fan JG, Shi JP, Wong VW, Eslam M, George J, et al. From NAFLD to MAFLD: a "redefining" moment for fatty liver disease. Chin Med J (Engl) 2020 Jul 21. doi: 10.1097/CM9.0000000000000981. [Epub ahead of print].

18. Lin S, Huang J, Wang M, Kumar R, Liu Y, Liu S, Wu Y, Wang X, Zhu Y. Comparison of MAFLD and NAFLD diagnostic criteria in real world. Liver Int 2020 Jun 1. doi: 10.1111/liv.14548. [Epub ahead of print].

19. Assante G, Williams R, Youngson NA. Is the increased risk for MAFLD 
patients to develop severe COVID-19 linked to perturbation of the gut-liver axis? J Hepatol 2020:S0168-8278(20)30391-30393.

20. Zhou YJ, Zheng KI, Wang XB, Yan HD, Sun QF, Pan KH, et al. Younger patients with MAFLD are at increased risk of severe COVID-19 illness: A multicenter preliminary analysis. J Hepatol 2020;73(3):719-721.

21. Zhou YJ, Zheng KI, Wang XB, Sun QF, Pan KH, Wang TY, et al. Metabolic-associated fatty liver disease is associated with severity of COVID-19. Liver Int 2020 Jun 23:10.1111/liv.14575. doi: 10.1111/liv.14575. [Epub ahead of print].

22. Lin S, Huang J, Wang M, Kumar R, Liu Y, Liu S, et al. Comparison of MAFLD and NAFLD diagnostic criteria in real world. Liver Int 2020 Jun 1. doi: 10.1111/liv.14548. [Epub ahead of print].

23. Fouad Y, Saad Z, Raheem EA, Moness H, Osman N, Abdelhameed W, et al. Clinical Validity of the diagnostic criteria for metabolic-associated fatty liver disease: a real-world experience. medRxiv 2020 doi: 10.1101/2020.08.20.20176214. [Epub ahead of print]. 